\title{
ARCHITECTING FARMER-CENTRIC INTERNET OF THINGS FOR PRECISION CROP PRODUCTION
}

\author{
Author(s):
}

A. Nyéki, G. Teschner, B. Ambrus, M. Neményi, A. J. Kovács

\section{Affiliation:}

Department of Biosystems and Food Engineering, Faculty of Agricultural and Food Sciences, Széchenyi István University, Vár tér 2., Mosonmagyaróvár, H-9200, Hungary

\section{Email address:}

nyeki.aniko@sze.hu; teschner.gergely@sze.hu; ambrus.balint@sze.hu; nemenyi.miklos@sze.hu; kovacs.attila@sze.hu

Abstract: The aim of this study is to develop a decision support system using sensors and IoT (Internet of Things)-based application in precision agriculture, first of all, in Hungary. These new technologies have the potential to transform decision-making in agriculture and to reduce environmental impact. In this paper, the advantages are demonstrated of two types of sensor sets that were installed to collect data from soil, plant, and the environment. Data are processed in a decision-making system. The proposed system allows users to monitor the fields in real-time and gives recommendations for production using wireless and internet communication.

Keywords: sensors, wireless network, LoRaWAN, precision agriculture, IoT

\section{Introduction}

Major IoT technologies include radio frequency identification technology, sensor technology, sensor network technology and internetwork communication, all of which have been involved in the four links of IoT industrial chain, namely, identification, sensing, processing and information delivery (TongFe, 2017). In this way, IoT can operate certain programs and realize remote control (Kovács and Husti, 2018). The Internet of Thinsg (IoT) provides lots of sensor data. However, the data by themselves do not provide value unless it is converted into some action. Real-time sensor data analysis and decision-making is often done manually but to make it scalable, it is preferably automated (Bandi et al., 2017). Smart farming era has already begun and its social and environmental impacts are expected to be major. Thought IoT technologies have became the large-scale path towards practices (Glaroudis et al., 2020). IoT has the potential to become the key enabler for realizing the vision of smart agriculture (Sinha et al., 2019). IoT are associated thorough Internet via Wireless Sensor Networks (WSN), Radio-frequency Identification (RFID), Bluetooth, Near-field communication (NFC), Long Term Evolution (LTE) and various other smart communication technologies (Khanna and Kaur, 2019).

IoT in this way some sensors connect the internet, so as to operate certain programs and realize remote control. The central computer can Smart Agriculture Based on Cloud Computing and IoT realize concentrated management and control of machine, equipment and personnel based on the internet and improve production and life through more detailed and dynamic means. This is useful for integration and harmony between human society and the physical world and is regarded as the third wave of information industry development following computer and internet. Major IoT technologies include radio frequency identification technology, sensor technology, sensor network technology and internetwork communication, all of which have been involved in the four links of IoT industrial chain, namely, identification,

SmartFarmNet IoT based platform is capable of automating the process of data collection from various parameters related to agriculture e.g. environmental, fertilization, soil, irrigation, etc. It is also capable of correlating data and filtering out invalid variable from the perspective of assessing crop performance and computation crop forecasts (Jayaraman et al., 2016).

The long-term aim is the continuous automatic validation of data provided by the on-the-go measuring systems that occurs while passing by the installed stations and making wireless corrections in the measurement of mobile devices. Based on previous background collected by research, the IoT system will be expanded and operated (Nyéki et al., 2020). The information, automatically gathered by the sensors, is 
analyzed and different techniques (e.g. decision support system and artificial intelligence) are compared. Artificial Intelligence (AI) provides the framework and tools to go beyond trivial real-time decision and automation use cases for IoT (Bandi et al., 2017). Until now, parameters were measured manually in a hard, long and expensive process. This new sensors network offers "Big Data" and clearer results.

\section{Internet of Things hardware, platforms and sensors in crop production}

The structure of IoT is based on three layers: namely, the perception layer (sensing), the network layer (data transfer) and the application layer (data storage and manipulation) (Tzounis et al., 2017).

The first expectation is a fault tolerant and energy efficient WSN architecture for real time monitoring of the field conditions. Several IoT based commercial platform have been developed with the vision of modernizing and enhancing the effectiveness of farming process (Sinha et al., 2019). Due to the distributed nature of IoT, in cases of battery-operated nodes, placed in crop fields or other agricultural facilities, replacing the power source can be a very difficult task, if not impossible. Other factors making a low-power, embedded device selectable for a deployment are its long-term stability, the number of digital and analogue inputs/outputs which determines the number of peripheral sensors (Tzounis et al., 2017).

The quality of field crops depends mainly the soil and the meteorological - seasonal- conditions they are growing. The soil type, chemical and physical conditions, moisture content and some other factors are responsible for the quality of crop yield. Information can be obtained using sensors, with low power devices that collect data from soil, crop and the surroundings by growing season. Sensing has led to adoption of technology in the precision agriculture and it also enables improved efficiency of agricultural production and practices (Vuran et al., 2018). To observe the growth of the crop under varying real-time conditions (e.g., soil quality, environmental conditions, etc.), typical crop studies involve phenotyping to understand the key factors (e.g., the soil pH level, the rate of nitrification) affecting crop growth (Jayaraman et al., 2016). The application of technology in the field are used to improve crop yield or quality and to reduce costs. The use of WSN in precision agriculture assists the farmers in a statistical number, helping them make better and well informed decisions (Fang et al., 2014; Kodali et al., 2014). Sensors are used for irrigation scheduling to measure humidity, solar radiation, temperature (Fourati et al., 2014).

Due to limited prosessing power and energy considerations, data processing, storage and decision making are not generally conducted locally. Field information can be stored in a private database (Yan et al., 2017). Sensor data is transmitted to a local gateway, which transit the database to data center for storage and analysis. Data center is mainly cloud or server storage. Real time monitoring by implementation of WSNs contributes to minization of potential production risks, emerging mainly from environmental influences and human actions (Wu et al., 2013). Furthermore, real time data from fields may be used by farmers to help adjust the crop production strategies at any time, without the need to use of a tractor to get to each sampling point. Therefore, the implementation of WSNs contributes to some extent to the decrease of energy consumption in agriculture (Srbinovska et al., 2015).

Table 1. Summary of the most popular IoT wireless technologies (Source: Tzounis et al., 2017)

\begin{tabular}{|c|c|c|c|c|c|c|}
\hline Wireless technology & Wireless standard & Network type & Operating frequency & Max. range & Max data rate \& power & Security \\
\hline Wifi & IEEE 802.11a, 11b, 11g, 11n, 11ac, 11ad & WLAN & $\begin{array}{l}2.4,3.6,5 \mathrm{GHz} \\
60 \mathrm{GHz}\end{array}$ & $100 \mathrm{~m}$ & $\begin{array}{l}\text { 6-780 Mbps } 6.75 \mathrm{Gbps} \text { at } 60 \mathrm{GHz} \\
1 \text { Watt }\end{array}$ & WEP, WPA, WPA2 \\
\hline Z-wave & Z-wave & Mesh & $908.42 \mathrm{MHz}$ & $30 \mathrm{~m}$ & $100 \mathrm{Kbps}, 1 \mathrm{~mW}$ & Triple DES \\
\hline Bluetooth & Bluetooth (Formerly IEEE 802.15.1) & WPAN & $2400-2483.5 \mathrm{MHz}$ & $100 \mathrm{~m}$ & $1-3 \mathrm{Mbps}, 1 \mathrm{~W}$ & $56 / 128$ bit \\
\hline 6LOWPAN & IEEE 802.15 .4 & WPAN & $908.42 \mathrm{MHz}$ or $2400-2483.5 \mathrm{MHz}$ & $100 \mathrm{~m}$ & $250 \mathrm{Kbps}, 1 \mathrm{~mW}$ & 128 bit \\
\hline Thread & IEEE 802.15.4 & WPAN & $2400-2483.5 \mathrm{MHz}$ & N/A & $\mathrm{N} / \mathrm{A}$ & N/A \\
\hline Sigfox & Sigfox & WPAN & $908.42 \mathrm{MHz}$ & $30-50 \mathrm{~km}$ & $10-1000 \mathrm{bps}$ & $\mathrm{N} / \mathrm{A}$ \\
\hline LORaWAN & LORaWAN & WPAN & Various & $2-15 \mathrm{~km}$ & $0.3-50 \mathrm{kbps}$ & $\mathrm{N} / \mathrm{A}$ \\
\hline BluetoothSmart (BLE) & loT Inter-connect & WPAN & $2400-2483.5 \mathrm{MHz}$ & $100 \mathrm{~m}$ & $1 \mathrm{Mbps}, 10-500 \mathrm{~mW}$ & 128pbit AES \\
\hline Zigbee & IEEE 802.15 .4 & Mesh & $2400-2483.5 \mathrm{MHz}$ & $10 \mathrm{~m}$ & $250 \mathrm{Kbps}, 1 \mathrm{~mW}$ & 128 bit \\
\hline THREAD & IEEE 802.15.4, 6LoWPAN & Mesh & $2400-2483.5 \mathrm{MHz}$ & $11 \mathrm{~m}$ & $251 \mathrm{Kbps}, 2 \mathrm{~mW}$ & 128 bit AES \\
\hline RFID & Many standards & Point to Point & $13.56 \mathrm{MHz}$ & $1 \mathrm{~m}$ & $423 \mathrm{Kbps}$, about $1 \mathrm{~mW}$ & Possible \\
\hline NFC & ISO/IEC 13157 & Point to Point & $13.56 \mathrm{MHz}$ & $0.1 \mathrm{~m}$ & $424 \mathrm{Kbps}, 1-2 \mathrm{~mW}$ & Possible \\
\hline GPRS & $3 \mathrm{GPP}$ & GERAN & GSM 850, $1900 \mathrm{MHz}$ & $25 \mathrm{~km} / 10 \mathrm{~km}$ & $\begin{array}{l}171 \mathrm{Kbps} \\
2 \mathrm{~W} / 1 \mathrm{~W}\end{array}$ & GEA2/GEA3/GEA4 \\
\hline EDGE & 3 GPP & GERAN & GSM 850/1900 MHz & $26 \mathrm{~km} / 10 \mathrm{~km}$ & $384 \mathrm{Kbps}, 3 \mathrm{~W} / 1 \mathrm{~W}$ & $\mathrm{~A} 5 / 4, \mathrm{~A} 5 / 3$ \\
\hline HSDPA/HSUPA & 3GPP & UTRAN & $850 / 1700 / 1900 \mathrm{MHz}$ & $27 \mathrm{~km} / 10 \mathrm{~km}$ & $0.73-56 \mathrm{Mbps}, 4 \mathrm{~W} / 1 \mathrm{~W}$ & USIM \\
\hline LTE & $3 \mathrm{GPP}$ & GERAN/UTRAN & $700-2600 \mathrm{MHz}$ & $28 \mathrm{~km} / 10 \mathrm{~km}$ & $0.1-1 \mathrm{Gbps}, 5 \mathrm{~W} / 1 \mathrm{~W}$ & $\begin{array}{l}\text { SNOW 3G } \\
\text { Stream Cipher }\end{array}$ \\
\hline ANT + & ANT + Alliance & WSN & $2.4 \mathrm{GHz}$ & $100 \mathrm{~m}$ & $1 \mathrm{Mbps}, 1 \mathrm{~mW}$ & AES- 128 \\
\hline Cognitive Radio & IEEE 802.22 WG & WRAN & $54-862 \mathrm{MHz}$ & $100 \mathrm{~km}$ & $24 \mathrm{Mbps}, 1 \mathrm{~W}$ & AES-GCM \\
\hline Weightless-N/W & Weightless SIG & LPWAN & $700 / 900 \mathrm{MHz}$ & $5 \mathrm{~km}$ & $0.001-10 \mathrm{Mbps}, 40 \mathrm{~mW} / 4 \mathrm{~W}$ & 128 bit \\
\hline
\end{tabular}


Numerous wireless devices have been developed upon the various wireless standards. As can be seen in Table 1, IoT wireless communications provide a wide variety of bandwidth, power consumption, network type and communication range. So, the number of sensor sets (or nodes), the distance between them, the operating frequency based on the size of messages (size of required data) are pivotal factors to be taken into consideration, when choosing a wireless transceiver for open-field operation.

LoRa (LongRange) is a digital wireless data communication technology used long-range connectivity for various IoT devices. The LoRaWAN network ensures the messages between end-devices (sensors) and a central network server. The major benefits of this system are low power, low range and low cost connectivity. LoRa has brought in a lot of interesting application such as utilities, precision agriculture and smart city (Singh et al., 2020).

\section{Materials and methods}

\section{Agro-IoT system in Mosonmagyaróvár (M-AIoT)}

The systems main purpose is to collect data from crop fields and from the surrounding natural (or quasinatural) areas. Thereby, the relationship between natural ecology and agro-ecology can be profoundly studied. One of the tools of processing Big Data is using artificial intelligence.

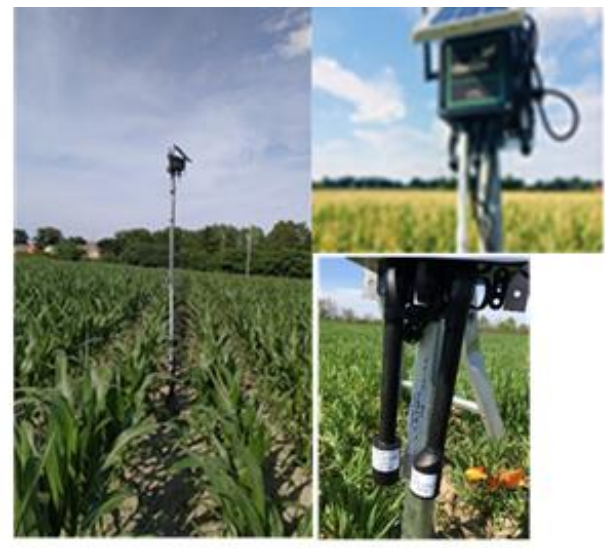

Installed sensors in a research crop field, measured parameters : air temperature, humidity, pressure, $\mathrm{CO}_{2}$ and ammonia
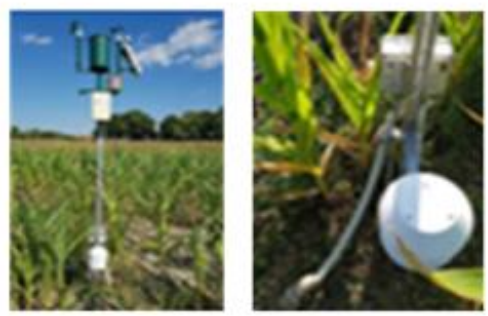

air temperature, humidity, wind speed, precipitation and global radiation

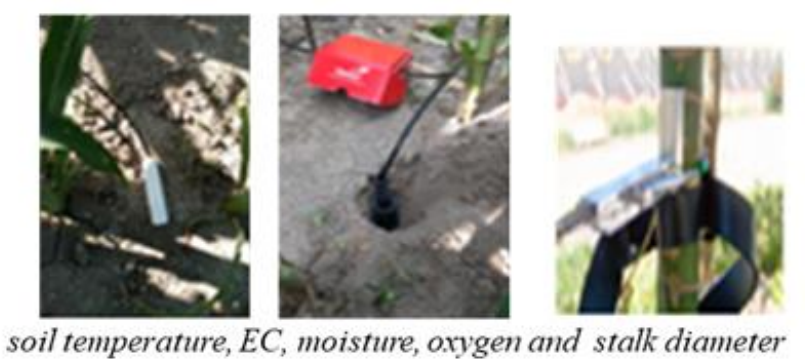

Figure 1. Sensor components of M-AIoT system in Mosonmagyaróvár Source: based on Nyéki et al., 2020 


\section{Components of M-AIoT system}

The system consists of soil, crop, environment and atmospherics sensors (Figure 1). This system is based on solar energy power. In this project Raspberry $\mathrm{Pi}$, Arduino and Libelium (http://www.libelium.com/) platforms.

\section{Results}

This paper presents an IoT web-based platform for agriculture farming system with the use of Raspberry Pi, Arduino and Libelium platforms. The results focuses on online monitoring of fields with LoRaWan communication protocol.

By interconnecting all the sensors shown above, we proposed a system for a commercial use for monitoring agricultural fields.

\section{Server structure}

In this project was designed a system structure which can integrate the database from different sensors (from different producer) using different communication protocols. The server development was implemented in .NET language. MS SQL database was used to store the sensor data. The system consists Raspberry Pi, Arduino platforms using different sensors with GSM communication, which were developed by research team (Figure 2). Arduino and Raspberry Pi platforms with GSM communication were developed in this project, which have different purpose and sensors. We also use Libelium's sensors and Lorawan's data communication device through purchases.

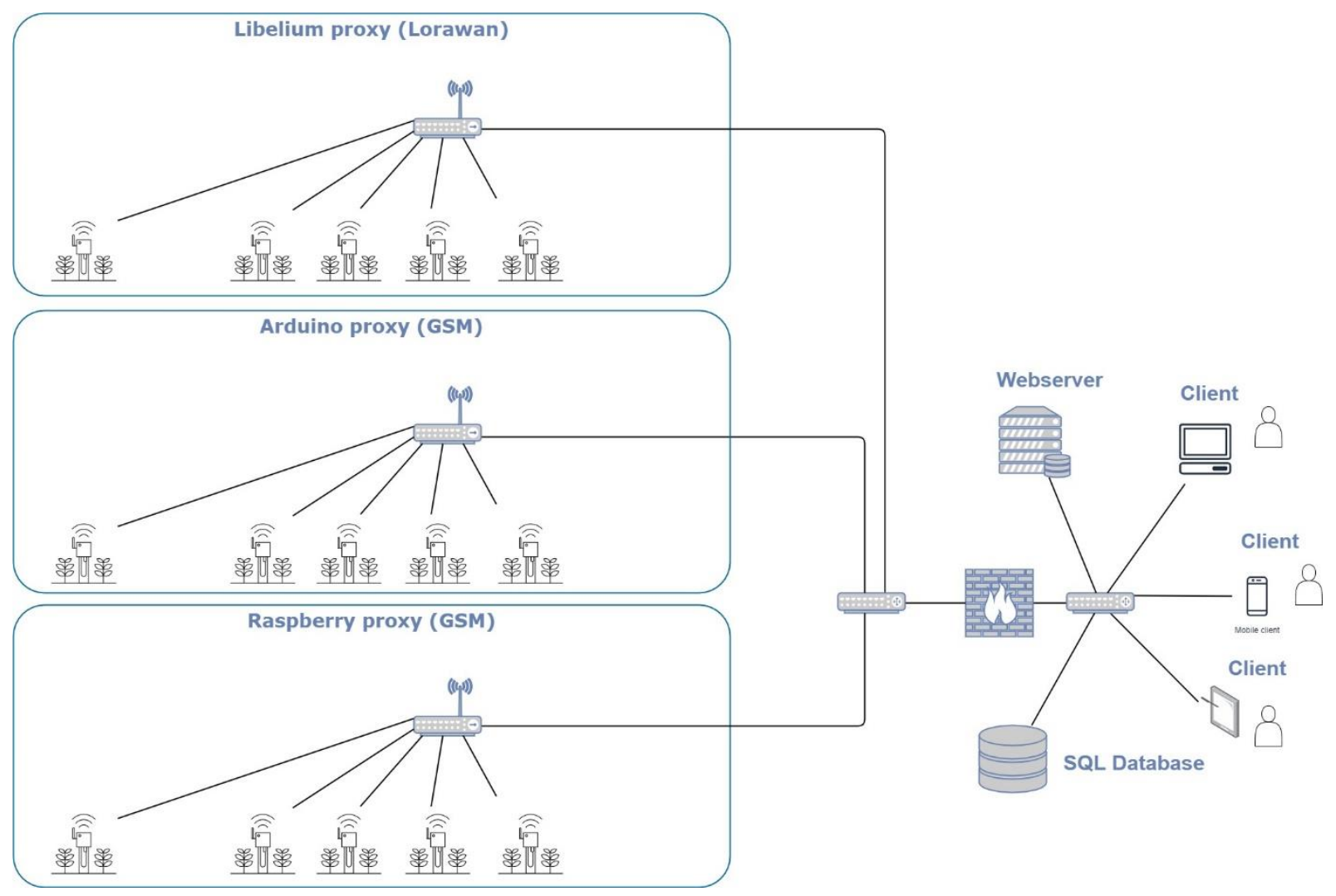

Figure 2. Diagram of IoT server structure

The aim of the IoT-based web interface development was a farmer-centric system that allows (displays) for end-users the clear database enhanced useful information for farming. Therefore, a web application (site) was development that already supports useful tools what was development in Angular platform. The web interface is designed to handle the different GPS-based measuring stations from crop fields uniformly. This 
web-based system can provide a comparative and valuable analysis with the experiences and conclusions. The analysis can help the farmers and makes them better decisions related to field management (e.g. optimal time for sowing, optimum nitrogen fertilizer amount, irrigation recommendation). The improvement was constructed in Angular framework. The development of system applications and tools are continuous.

The achieved tools and features of system are the following.

As web administrator:

- adding a new sensor types to the system,

- application of new sensors with IDs, units, measuring ranges,

- register a new sensor station with GPS coordinates,

- register a new repeaters in the system.

As user:

- viewing measurement data,

- tabular view,

- chart view,

- handling alerts,

- overviewing of sensor (measuring) stations on a map.

Figure 3 shows the dashboard with graphical display of values from field sensor. The user can choose the time interval and the sensor type from given GPS-based measuring station.

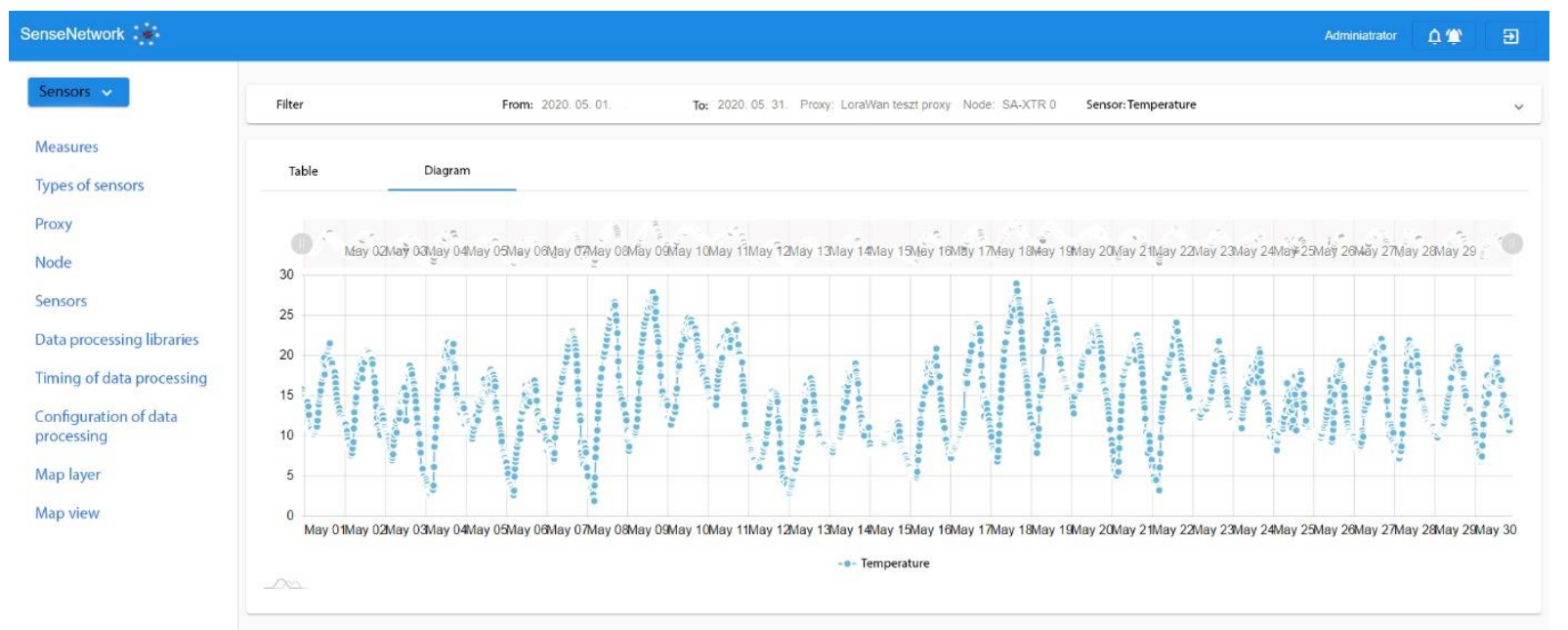

Figure 3. Graphical display of temperature values from the sensor

Figure 4 shows the alarm view of the user interface where the user can configure email and sms alerts. The complexity and intricacy of alarms can be adjusted to the specific microclimatic environment, crop or sensor. It is possible to examine the absolute value of a given sensor value. For example, if the soil temperature has reached 7 degrees Celsius. It is possible to examine the average of a given time interval, for example, the average air humidity over the past 12 hours was higher than $80 \%$. Furthermore, there is an opportunity for combining the conditions of different sensor data, e.g. if the air temperature in the last 24 hours has been higher than 15 degrees Celsius and the air humidity has been higher than $75 \%$ in the last 18 hours.

The specific alarms can be saved by the farmer. In the case of a certain condition or conditions system is confirmed, the farmer will be notified by e-mail and / or sms.

Additionally, through the platform, we can collect data from field sensors, such as temperature, dielectrical permittivity and soil electrical conductivity (Figure 5). These values are suitable, for example, for irrigation support.

The application has a high usable visual functionality on the basis of the variables acquired by the sensors. In this way, intelligent programming can be achieved for farmers, too, with simple programming settings. The field information come from soil, weather, environment and plant conditions. The essential web platform requirement in our project is to be able to store, scale, process of several hundreds of agricultural IoT sensors. 


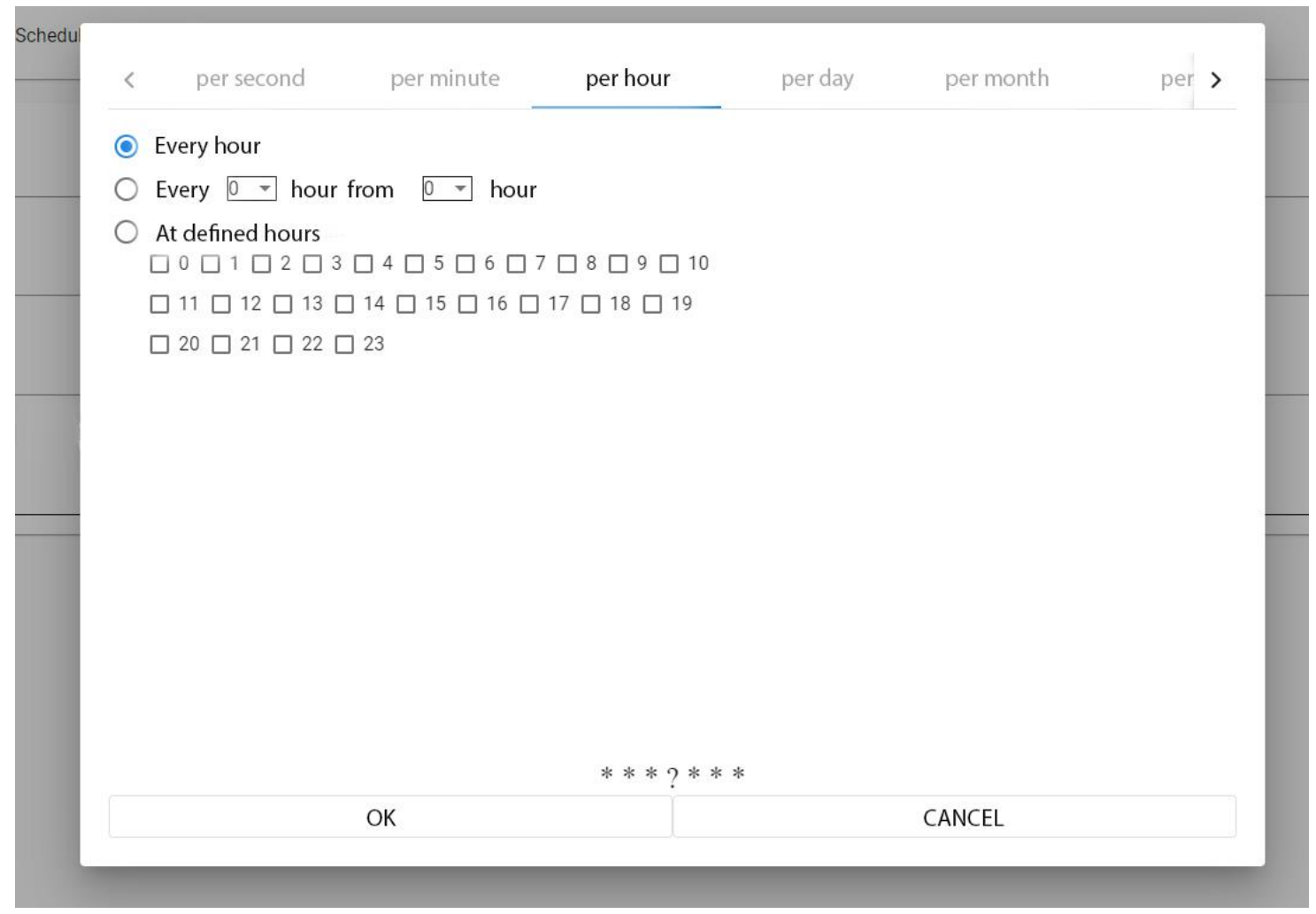

Figure 4. Specific alarms setup on IoT platform
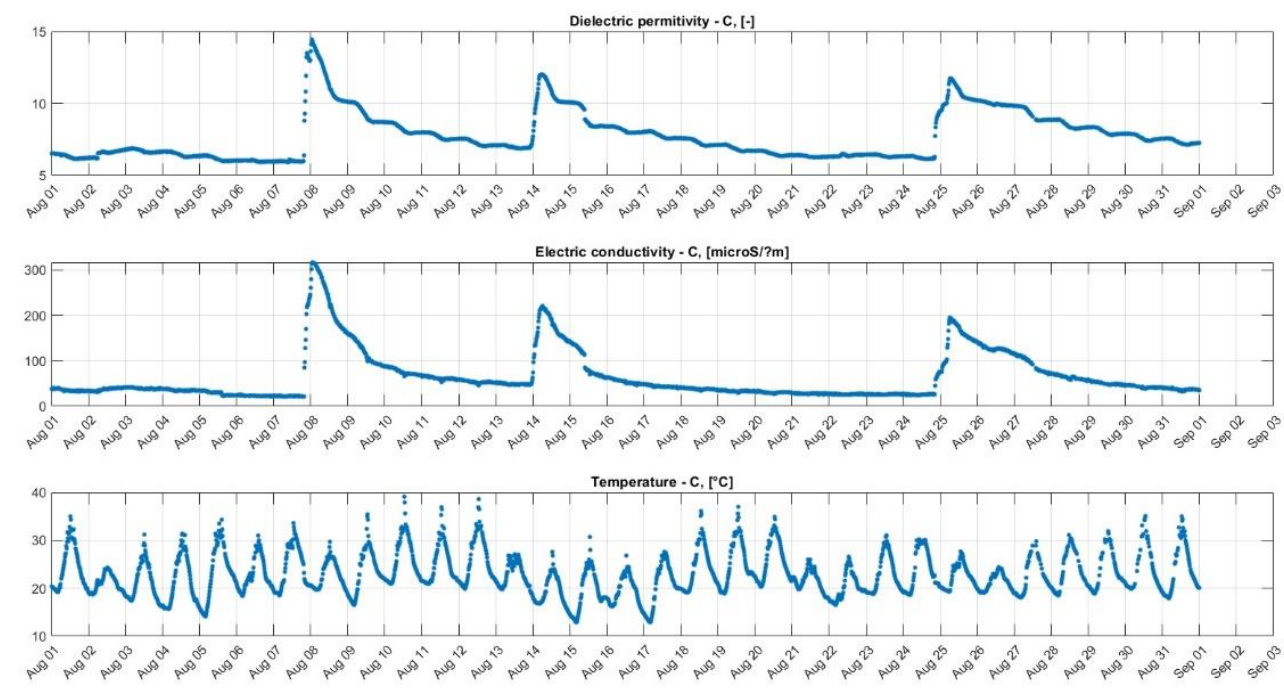

Figure 5. Dielectric permittivity, electrical conductivity and temperature measured from August 1 to September 3

\section{Conclusions}

In this paper, we proposed an IoT-based farmer-centric system, which provide automated control of crop production. The proposed system is beneficial for farmers as well as decision makers. The web-based system provides interconnections between different sensors using wireless and web technologies to monitor field conditions. In this paper have presented a new decision support system for agriculture using different sensors and connection types. This system comprises automatic sensors with wireless networks and web application. 
The database system will be implemented as a web-based system. The collected and stored data will be used for decision making to monitor and control the automatic management of crops. The system can send notifications about water demand of crop, temperature and moisture content of soil in order to suggest the irrigation, planting or other application. The user can also be used to determine the optimum time to crop protection. Moreover, this database allow data mining to analyze the data for predicting environmental variables for optimal future crop production management. Furthermore, we would like to implement effective prediction of sensors database using artificial intelligence. Therefore, the farmer will be able to manage more efficiently the site-specific, precision farming.

\section{Acknowledgement}

The project is financed by the "Thematic Area Excellence Program" of the National Research, Development and Innovation Office (TUDFO/51757/2019-ITM). The research was partly supported by the European Union and the Hungarian Government from the project 'FIEK - Center for cooperation between higher education and the industries at Széchenyi István University under grant number GINOP-2.3.4-15-201600003 .

\section{References}

[1] Sinha, A., Shrivastava, G., Kumar, P.: 2019. Architecting user-centric internet of things for smart agriculture. Sustainable Computing: Informatics and Systems. Vol. 23. 88-102 pp. https://doi.org/10.1016/j.suscom.2019.07.001

[2] Khanna, A., Kaur, S.: 2019. Evolution of Internet of Things (IoT) and its significant impact in the field of Precision Agriculture. Computers and Electronics. Vol. 157. 218-231 pp. https://doi.org/10.1016/j.compag.2018.12.039

[3] Jayaraman, P.P., Yavari, A., Georgakopoulos, D., Morshed, A., Zaslavsky, A..: 2016. Internet of Things platform for smart farming: experiences and lessons learnt. Sensors. Vol. 16. No. 11, 1884. https://doi.org/10.3390/s16111884

[4] Glaroudis, D., Iossifides, A., Chatzimisios, P.: 2020. Survey, comparison and research challenges of IoT application protocols for smart farming. Computer Networks. Vol. 168. https://doi.org/10.1016/j.comnet.2019.107037

[5] http://www.libelium.com/

[6] Nyéki, A., Neményi, M., Teschner, G., Milics, G., Kovács, A.J.: 2020. Application possibilities and benefits of IoT (Internet of Things) in agricultural practice. Quo vadis IoT? Hungarian Agricultural Engineering. Vol. 37. pp. 90-96. http://doi.org/10.17676/HAE.2020.37.90

[7] Bandi, R., Swamy, S., Raghav, S.: 2017. A framework to improve crop yield in smart agriculture using IoT. International Journal of Research in Science \& Engineering. Vol. 3. Issue: 1. 176-180 pp.

[8] Vuran, M.C., Salam, A., Wong, R., Irmak, S.: 2018. Architecture and technology aspects. Ad Hoc Networks. Vol. 81. 160-173 pp. https://doi.org/10.1016/j.adhoc.2018.07.017

[9] Yan, Q., Yang, H., Vuran, M.C., Irmak, S.: 2017. Scalable and private continual geo-distance evaluation for precision agriculture. In IEEE Conference on Communications and Network Security (IEEE CNS), Las Vegas, NV, USA.

[10] Tzounis, A., Katsoulas, N., Bartzanas, T., Kittas, C.: 2017. Internet of Things in agriculture, recent advances and future challenges. Biosystems Engineering. Vol. 164. 31-48 pp. https://doi.org/10.1016/j.biosystemseng.2017.09.007

[11] Fang, S., Da Xu, L., Zhu, Y., Ahati, J., Pei, H., Yan, J., Liu, Z.: 2014. An integrated system for regional environmental monitoring and management based on internet of things. IEEE Trans. Ind. Inform. Vol. 10. 1596-1605 pp.

[12] Kodali, R.K., Rawat, N., Boppana, L.: 2014. WSN sensors for precision agriculture. In: Region 10 Symposium, IEEE. 651-656 pp. https://doi.org/10.1109/TENCONSpring.2014.686311

[13] Singh, R.K., Berkvens, R., Weyn, M.: 2020. Synchronization and efficient channel hopping for power efficiency in LoRa networks: A comprehensive study. Internet of Things. Vol.11. 1-15 pp. https://doi.org/10.1016/j.iot.2020.100233

[14] Wu, D.D., Olson, D.L., Birge, J.R.: 2013. Risk management in cleaner production. Journal of Cleaner Production. Vol. 53. 1-6 pp. https://doi.org/10.1016/j.jclepro.2013.02.014 
[15] Srbinovska, M., Gavrosvski, C., Dimcev, V., Krkoleva, A.: 2015. Environmental parameters monitoring in precision agriculture using wireless sensor networks. Journal of Cleaner Production. Vol. 88. 297-307 pp. http://dx.doi.org/10.1016/j.jclepro.2014.04.036

[16] TongKe F.: 2017. Smart Agriculture Based on Cloud Computing and IOT. Journal of Convergence Information Technology, Vol. 8. No. 2. pp. 210-216. http://dx.doi.org/10.4156/jcit.vol8.issue2.26

[17] Kovács, I., Husti, I.: 2018. The role of digitalization in the agricultural 4.0 - How to connect the industry 4.0 to agriculture? Hungarian Agricultural Engineering. Vol. 33. 38-42 pp. https://doi.org/10.17676/HAE.2018.33.38 